\section{Passivrauchen fördert die Entwicklung atopischer Erkrankungen}

\author{
Passivrauchen ist in vielerlei Hinsicht schädlich. Dass sich dadurch bei \\ Kindern auch das Risiko für Nahrungsmittelallergien und Ekzeme erhöht, \\ kann nun eine Studie aus Schweden belegen.
}

Ü ber die ausführlichen Daten einer Langzeitstudie (die longitudinale Geburtskohortenstudie BAMSE) wollten Stockholmer Allergologen und Umweltmediziner abklären, ob Passivrauchen in utero und nach der Geburt ein Risiko für Sensibilisierungen beziehungsweise Allergien bei Kindern und Jugendlichen bis zum 17. Lebensjahr darstellt. Es konnten 3.316 Kinder der BAMSE-Kohorte untersucht werden. Die Wissenschaftler setzten zum einen wiederholt Fragebögen über die Exposition mit Zigarettenrauch und allergische Symptome ein, zum anderen fand eine ImmunoCAP-Bestimmung von spezifischem IgE gegen sechs gängige Nahrungsmittel- und acht Aeroallergene statt. Die Untersuchungen fanden jeweils im Alter von 4, 8 und 16 Jahren statt.

Passivrauchen im Mutterleib sowie in der frühen Kindheit erhöhte das Risiko für eine Sensibilisierung gegen Nahrungsmittel im Alter von 4, 8 und 16 Jahren. Die Odds Ratio [OR] lag bei den 4-Jährigen bei 1,47 bei einem 95\%-Konfidenzintervall [KI] von 1,08-2,00 und betrug über die gesamte Zeitspanne 1,24 bei einem 95\%-KI von 0,98-1,56.

Außerdem erwies sich eine Zigarettenrauchexposition als genereller Risikofaktor für eine Sensibilisierung plus ato- pischem Ekzem mit einer OR von 1,62 (95\%-KI: 1,20-2,18).

Darüber hinaus gab es eine dosisabhängige Assoziation mit einer ansteigenden Anzahl der Zigaretten, die der Vater zuhause rauchte. Dagegen beeinflusste etwas überraschend - das mütterliche Rauchen während der Schwangerschaft alleine nicht die jeweilige Atopieneigung der Kinder über alle genannten Altersstufen hinweg. Vermutlich hat Nikotin während der Schwangerschaft auch einen immunsuppressiven Effekt.

Thacher JD et al. Parental smoking and development of allergic sensitization from birth to adolescene. Allergy 2016;71:239-48

\section{Kommentar}

Müssen Kinder im elterlichen Milieu passiv rauchen, führt dies zu einem erhöhten Risiko einer Sensibilisierung gegen wichtige Nahrungsmittel und darüber hinaus auch zu einem erhöhten Risiko für eine allgemeine Sensibilisierung, verbunden mit einem atopischen Ekzem. Im Gegensatz zur deutschen Multicenter-Allergie-Studie MAS waren in Schweden diese Zahlen unabhängig von einem familiären Atopierisiko. Beeindruckend war hier die hohe Anzahl der Probanden und die lange Laufzeit der Studie sowie die Menge an Labordaten.

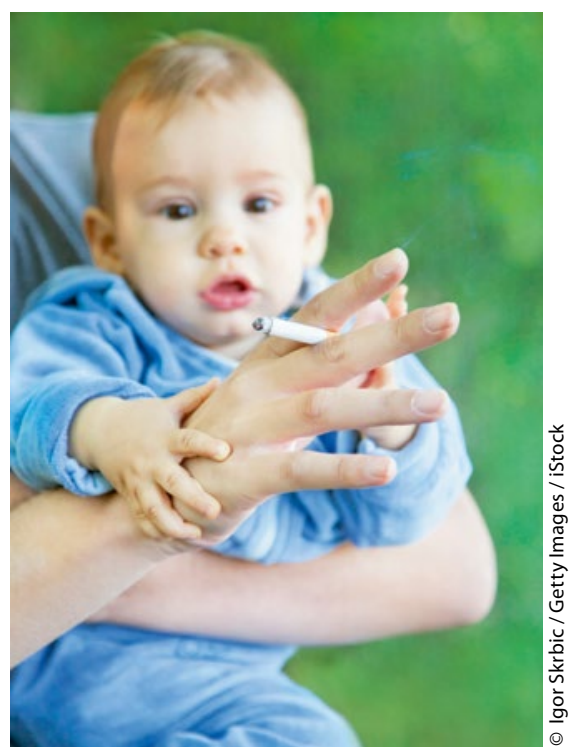

Passivrauchen in der frühen Kindheit erhöht das Risiko allergischer Sensibilisierungen.

Passivrauchen gilt somit als Risikofaktor für die Entwicklung atopischer Erkrankungen bei jungen und älteren Kindern. Ätiologische Erklärungen hierfür fehlen allerdings weitgehend. Das "Mucosal Concept of Atopy" vermutet, dass eine Sensibilisierung hauptsächlich in den Schleimhäuten der Atemwege stattfindet, indem eine Inflammation vor Ort die Antigenpenetration erleichtert. Und Allergene von Nahrungsmitteln finden sich öfters im Staub oder werden von Kleinkindern beim Essen aspiriert. Möglicherweise führt Tabakrauch auch zu negativen Effekten an der Hautbarriere und verstärkt dort das Eindringen von Nahrungsmittelallergenen.

Dr. Ulrich Mutschler 